\title{
Outbreaks of highly pathogenic porcine reproductive and respiratory syndrome in Jiangxi province, China
}

Aijiang Guo, Guohua Wu, Wei Gong, Xuenong Luo, Haixue Zheng, Huanjie Jia and Xuepeng Cai ${ }^{*}$

\begin{abstract}
In 2007, herds of pigs in Jiangxi Province, China experienced outbreaks of a severe form of suspected porcine reproductive and respiratory syndrome (PRRS) characterized by high fever, high morbidity and mortality in animals of different ages. 152 swine sera and 42 tissues (consisting of liver, lung, lymph node and kidney) from five herds of pigs were collected. Pigs were diagnosed as infected with a highly pathogenic form of the PRRS virus (PRRSV) based on ELISA and reverse transcriptase polymerase chain reaction (RT-PCR) results. Serological surveys indicated that $67-100 \%$ of the examined pig herds in Jiangxi Province were seropositive. 42 tissue samples were used to detect classical swine fever virus, porcine circovirus type 2 and PRRSV. Results indicated that only PRRSV was detected in 42 samples. 12 PRRSV amplified products of five herds, which consisted of two or three samples randomly selected from each herd, were used for sequencing. Subsequent nucleotide sequencing showed that the NSP2 gene had 99-99.7\% nucleotide and 99.2-100\% derived amino acid sequence identities among 12 tissues with that of the PRRS-JXA1 strain, deletions of 29 amino acids corresponded to positions 534-562 of the NSP2 gene sequence. These results revealed that the diseased pigs were all caused by fatal PRRSV variant. Compared with the same period in 2006, the number of positive cases from Jiangxi Province remained unchanged. These findings demonstrated that the highly pathogenic Northern American type PRRSV was still spreading in Jiangxi Province, China in 2007.
\end{abstract}

Keywords: Highly pathogenic, PRRS, ELISA, RT-PCR, NSP2, Swine herds

\section{Background}

Porcine reproductive and respiratory syndrome (PRRS) is caused by the porcine reproductive and respiratory syndrome virus (PRRSV). This virus was first isolated in 1991 and later identified as a small enveloped virus with a polyadenylated RNA genome, which contains eight open reading frames (ORFs) of approximately $15 \mathrm{~kb}$ in length $[1,2]$. PRRSV is considered to be one of the most economically important pathogens of pigs [3-7]. PRRSV strains have been grouped into European (Lelystad) types and North American (GenBank: VR2332) on the basis of genetic and antigenic differences between the

\footnotetext{
* Correspondence: caixp@vip.163.com

State Key Laboratory of Veterinary Etiological Biology, National Foot and Mouth Disease Reference Laboratory, Gansu Provincial Engineering and Technique Research Centre on Biological Detection, Lanzhou Veterinary Research Institute, Chinese Academy of Agricultural Sciences, 1 Xujiaping Yanchangbu Chengguan District, Lanzhou 730046, China
}

isolates from the two continents [8]. The JXA1 isolate belongs to the North American PRRSV genotype by phylogenetic analysis based on the nucleotide sequence of the structural protein ORFs [9]. Although different PRRSV strains cause similar diseases in pigs, previous studies have shown that the antigenicity and pathogenicity vary substantially among PRRSV strains identified around the world [10-15]. Many studies have shown that the non-structural protein 2 (NSP2)-coding gene (nsp2) in the PRRSV genome may represent the most genetically variable region [16-19].

HB-2 exhibited variations in the NSP2 nonstructural protein with a deletion of 12 amino acids in comparison with other North American PRRSV isolates [5]. A 29 aa deletion in the NSP2 protein of JXA1 occurs from amino acid positions 534 to 562 [9], which could be used as a molecular hallmark. PCR-based assays showed that many molecular markers for PRRSV could be amplified

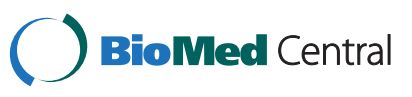


using primers specific for unique gene fragments of PRRSV [20-22].

Here, we report the outbreaks of highly pathogenic PRRSV in five herds of pigs in Jiangxi Province, China. Using the IDEXX PRRS ELISA serology test kit we examined sera, and the RT-PCR technique was used to amplify the NSP2 segments from the samples of deceased pig lung to obtain further molecular evidence.

\section{Case presentation \\ Methods \\ Serum and tissues}

No PRRSV vaccination had been carried out on any of the pig herds. Sera and tissue samples of lymph nodes, lungs, livers, and kidneys from diseased pigs suspected of being infected with PRRSV were collected in 2007 from pig herds located in Jiangxi Province, China, where pigs had high morbidity and mortality rates.

The North American PRRSV strain JXA1, isolated in our laboratory, and the attenuated vaccine strain Ch-1a (GenBank: AY032626) saved by our lab were used as positive controls. Pigs that were bred in Laboratory Animal Center of Lanzhou Veterinary Research Institute which did not show any clinical symptoms or gross lesions were used as negative control pigs. All animals were handled in strict accordance with good animal practice according to the Animal Ethics Procedures and Guidelines of the People's Republic of China, and the study was approved by the Animal Ethics Committee of Lanzhou Veterinary Research Institute, Chinese Academy of Agricultural Sciences (No. LVRIAEC-2010-002).

\section{Enzyme-linked immunosorbent assay (ELISA)}

Antibody titers were determined using a commercially available ELISA kit (IDEXX HerdCheck PRRS, Westbrook, Maine). According to the manufacturer's instructions, tested serum samples for which the optical density ratio is $\geq 0.4$ were considered to be positive against PRRSV.

\section{RNA extraction and RT-PCR}

42 frozen clinical samples were homogenized for 15-30 s with a homogenizer [23]. Viral genomic RNA was extracted simultaneously from homogenized tissues and from lysates of infected cell cultures (positive control) with the TaKaRa MiniBEST Viral RNA Extraction Kit Ver.3.0 according to the manufacturer's protocol (TaKaRa, Dalian, Japan). RT-PCR of partial Nsp2 was performed using TaKaRa One Step RT-PCR kit (TaKaRa, Dalian, Japan) following the manufacturer's instructions. Comparisons of the whole genome sequence of four variants $(\mathrm{HeB}, \mathrm{HuB}$, JXA1, and HuB1, GenBank accession numbers EF112447, EF112446, EF112445, and EF075945, respectively), and the Northern American strain VR2332
(GenBank accession number AY150564), sequence of the nucleotide deletion region (30 amino-acid deletion in Nsp2 of highly pathogenic PRRS) and the conserved region in Nsp2 segments were obtained. The nucleotide deletion region was used as molecular hallmark. Specific primers for PRRSV were used to amplify the partial Nsp2 segments. The primer pairs NSP2-F (5'- TGG GCA GCC GAG CAG GTT GAT TTA -3') and NSP2-R (5'- GTG GGG CGG CGG TGT CTC G -3') were used to generate an expected 855-bp fragment for the deletion form or a 945-bp fragment for the non-deletion form of PRRSV strains (from nt 2566 to 3420 of the JXA1 strain, GenBank accession number EF112445, and from nt 2567 to 3511 of the $\mathrm{CH}-1 \mathrm{a}$ strain, GenBank accession AY032626). Meanwhile swine fever virus and porcine circovirus type 2 were also detected by RT-PCR and PCR Technique according published methods $[24,25]$.

\section{Nucleotide sequencing}

12 amplified products of five herds, which consisted of two or three samples randomly selected from each herd, were purified using a PCR purification kit (Qiagen, Germany) and cloned using the pGEM-T easy Vector System I (Promega, USA) according to the manufacturer's instructions. The target fragments were sequenced using a Big Dye Terminator sequencing kit on an ABI 3730 DNA sequencer (Applied Biosystems, USA).

\section{Results and discussion \\ Clinical signs}

In the five affected swine herds, disease was characterized by a prolonged high fever of above $41^{\circ} \mathrm{C}$, inappetence and clear lethargy, followed by the development of respiratory distress, nervous signs, red discoloration in skin, and cyanopathy in the ears (Figure 1A). Lactating sows and piglets fell ill first. The main clinical signs in lactating sows were fever, anorexia, decrease of milk yield and nasal discharge. Few lactating sows had nervous signs. Piglets mainly exhibited high and continuous fever (over $41^{\circ} \mathrm{C}$ ), and nervous signs. Weanling and grower pigs became sick later and the clinical signs included continuous fever. The body and ears, and intermittent nervous signs. Nervous signs included incoordination and the adoption of unusual stances, which quickly progressed to opisthotonus and an inability to stand After 10-15 days, the disease quickly spread throughout each herd, lasting one month. The average mortality rates recorded on the five farms was $51 \%$ in boars, $72 \%$ in sows, and more than $85 \%$ in suckling piglets and nursery pigs (Table 1).

\section{Pathological changes}

The diseased pigs which displayed clinical signs, as mentioned above, were subjected to necropsy examinations. 


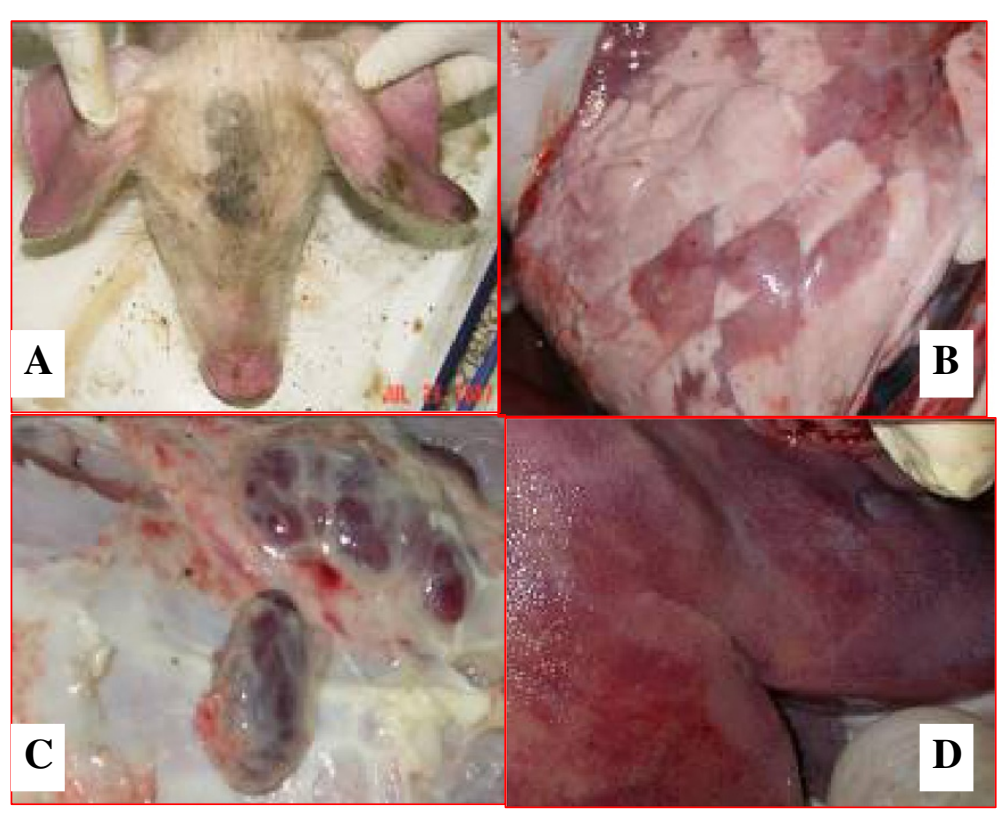

Figure 1 Clinical signs and pathological changes in pigs caused by PRRSV.

The common lesions included interstitial pneumonia (Figure 1B) associated with hemorrhage and fluid exudation, swollen haemorrhagic lymph nodes (Figure 1C), bronchial and hemorrhage, large number of hemorragic areas in kidney and liver (Figure 1D), intestine ulceration, gastric hemorrhage as well as meneaingeal hyperaemia hemorrhage in meninges. The lymph follicles, spleens, and kidneys had severe histopathologic changes.

\section{Screening of swine sera by ELISA}

Serological tests indicated that $67-100 \%$ of these pig herds in Jiangxi Province were PRRSV seropositive (Table 2).

\section{Detection of classical swine fever virus, porcine circovirus} type 2 and PRRSV

In this study, positive controls and 42 tissues from five pig herds experiencing severe disease outbreaks in 2007 were all positive for PRRSV, and negative for classical swine fever virus and porcine circovirus type 2. Control

Table 1 Mortality in pig herds caused by PRRSV according to age and sex

\begin{tabular}{llll}
\hline Herds & \multicolumn{2}{l}{ Dead no./total no. } \\
\cline { 2 - 4 } & Boars & Sows & $\begin{array}{l}\text { Suckling piglets and } \\
\text { nursery pigs }\end{array}$ \\
\hline 1 & $4 / 8$ & $36 / 48$ & $34 / 36$ \\
\hline 2 & $3 / 7$ & $22 / 33$ & $32 / 38$ \\
\hline 3 & $6 / 11$ & $51 / 67$ & $44 / 51$ \\
\hline 4 & $2 / 5$ & $14 / 18$ & $28 / 34$ \\
\hline 5 & $4 / 6$ & $29 / 36$ & $17 / 22$ \\
\hline Mean & $19 / 37=51 \%$ & $152 / 202=72 \%$ & $155 / 181=85.6 \%$ \\
\hline
\end{tabular}

pigs did not show any clinical signs or gross lesions, and lungs were negative for PRRSV by RT-PCR. Subsequent sequencing analysis of 12 samples revealed a 29 -aa deletion corresponding to positions 534-562 of Nsp2. They shared $99.2-100 \%$ identity in the predicted amino acid sequence with strain JXA1 isolated in 2006, which had deletions of one and 29 amino acids relative to the Nsp2 protein of VR-2332. The results showed that the emerging PRRSV, characterized by deletions in Nsp2, is highly pathogenic to pigs. The first outbreaks of highly pathogenic porcine reproductive and respiratory syndrome virus in China occurred in 2006 [9,26]. Compared with the same period in 2006 [9,22], the number of positive cases from Jiangxi Province in 2007 remained unchanged.

\section{Conclusions}

These pig herds were diagnosed as being infected with a highly pathogenic type of North American PRRSV, suggesting it was widespread in Jiangxi Province, China. The results of the present study provide base-line

Table 2 Seroprevalence of antibodies against PRRSV in pigs in Jiangxi Province, China in 2007

\begin{tabular}{lcc}
\hline Herds & No. of positive sera & No. of sera examined \\
\hline 1 & 10 & 10 \\
\hline 2 & 10 & 10 \\
\hline 3 & 14 & 16 \\
\hline 4 & 17 & 24 \\
\hline 5 & 62 & 92 \\
\hline Total & 113 & 152 \\
\hline
\end{tabular}


information for further epidemiological survey and control of highly pathogenic PRRSV in China.

\section{Abbreviations}

PRRS: Porcine reproductive and respiratory syndrome; ELISA: Enzyme-linked immunosorbent assay; ORFs: Open reading frames.

\section{Competing interests}

The authors declare that they have no competing interests.

\section{Authors' contributions}

AG wrote the manuscript; $A G, H Z$ and GW performed sample collection, RNA extraction and RT-PCR; WG and XH performed ELISA of serum; XL participated in sequence analysis; $\mathrm{HJ}$ detected classical swine fever virus and porcine circovirus type 2 of the samples; XC and AG were involved in the revision of the manuscript, the project design and the analysis and interpretation of the data assembled in this work. All authors read and approved the final manuscript.

\section{Acknowledgements}

We are grateful to Professor X. Q. Zhu of Lanzhou Veterinary Research Institute, Chinese Academy of Agricultural Sciences for improving the quality of the manuscript. The authors also thank the Key Scientific and Technology Program of Lanzhou, Gansu, China (No. 2008-1-180) for funding this investigation.

\section{Author details}

State Key Laboratory of Veterinary Etiological Biology, National Foot and Mouth Disease Reference Laboratory, Gansu Provincial Engineering and Technique Research Centre on Biological Detection, Lanzhou Veterinary Research Institute, Chinese Academy of Agricultural Sciences, 1 Xujiaping Yanchangbu Chengguan District, Lanzhou 730046, China.

Received: 30 December 2011 Accepted: 25 June 2012 Published: 11 July 2012

\section{References}

1. Wensvoort G, Terpstra C, Pol JM, Ter Laak EA, Bloemraad M, De Kluyver EP, Kragten C, Van Buiten L, Den Besten A, Wagenaar F: Mystery swine disease in The Netherlands: the isolation of Lelystad virus. Vet Q 1991, 13:121-130.

2. Meulenberg JJ, Hulst MM, de Meijer EJ, Moonen PJM, den Besten A, De Kluyver EP, Wensvoort G, Moormann RJ: Lelystad virus, the causative agent of porcine epidemic abortion and respiratory syndrome (PEARS), is related to LDV and EAV. Virology 1993, 206:62-72.

3. Forsberg R, Oleksiewicz MB, Petersen AM, Hein J, Botner A, Storgaard T: A molecular clock dates the common ancestor of European-type porcine reproductive and respiratory syndrome virus at more than $10 \mathrm{y}$ before the emergence of disease. Virology 2001, 289:174-179.

4. Plagemann PG: Porcine reproductive and respiratory syndrome virus: Origin hypothesis. Emerg Infect Dis 2003, 9:903-908.

5. Gao ZQ, Guo X, Yang HC: Genomic characterization of two Chinese isolates of porcine respiratory and reproductive syndrome virus. Arch Virol 2004, 149:1341-1351.

6. Ropp SL, Wees CE, Fang Y, Nelson EA, Rossow KD, Bien M, Arndt B, Preszler S, Steen P, Christopher-Hennings J, Collins JE, Benfield DA, Faaberg KS: Characterization of emerging European-like porcine reproductive and respiratory syndrome virus isolates in the United States. J Virol 2004, 78:3684-3703.

7. Thanawongnuwech R, Amonsin A, Tatsanakit A, Damrongwatanapokin S: Genetics and geographical variation of porcine reproductive and respiratory syndrome virus (PRRSV) in Thailand. Vet Microbiol 2004, 101:9-21.

8. Meng XJ, Paul PS, Halbur PG, Lum MA: Phylogenetic analyses of the putative $\mathrm{M}(\mathrm{ORF} 6)$ and $\mathrm{N}$ (ORF 7) genes of porcine reproductive and respiratory syndrome virus (PRRSV): Implication for the existence of two genotypes of PRRSV in the U.S.A. and Europe. Arch Virol 1995, 140:745-755.

9. Tian KG, Yu XL, Zhao TZ, Feng YJ, Cao Z, Wang CB, Hu Y, Chen XZ, Hu DM, Tian XS, Liu D, Zhang S, Deng XY, Ding YQ, Yang L, Zhang YX, Xiao HX,
Qiao MM, Wang B, Hou LL, Wang XY, Yang XY, Kang LP, Sun M, Jin P, Wang SJ, Kitamura Y, Yan JH, Gao GF: Emergence of fatal PRRSV variants: unparalleled outbreaks of atypical PRRS in China and molecular dissection of the unique hallmark. PLoS One 2007, 2(6):e526.

10. Meng XJ: Heterogeneity of porcine reproductive and respiratory syndrome virus: implications for current vaccine efficacy and future vaccine development. Vet Microbiol 2000, 74:309-329.

11. Pesente P, Rebonato V, Sandri G, Giovanardi D, Ruffoni LS, Torriani S: Phylogenetic analysis of ORF5 and ORF7 sequences of porcine reproductive and respiratory syndrome virus (PRRSV) from PRRS-positive Italian farms: a showcase for PRRSV epidemiology and its consequences on farm management. Vet Microbiol 2006, 114:214-224.

12. Yoshii M, Kaku Y, Murakami Y, Shimizu M, Kato K, Ikeda H: Genetic variation and geographic distribution of porcine reproductive and respiratory syndrome virus in Japan. Arch Virol 2005, 150:2313-2324.

13. Yuan S, Murtaugh MP, Schumann FA, Mickelson D, Faaberg KS: Characterization of heteroclite subgenomic RNAs associated with PRRSV infection. Virus Res 2004, 105:75-87.

14. Chen J, Liu T, Zhu CG, Jin YF, Zhang YZ: Genetic variation of Chinese PRRSV strains based on ORF5 sequence. Biochem Genet 2006, 44:425-435.

15. An TQ, Zhou YJ, Liu GQ, Tian ZJ, Li J, Qui HJ, Tong GZ: Genetic diversity and phylogenetic analysis of glycoprotein 5 of PRRSV isolates in mainland China from 1996 to 2006: coexistence of two NAsubgenotypes with great diversity. Vet Microbiol 2007, 123:43-52.

16. Allende R, Lewis TL, Lu Z, Rock DL, Kutish GF, Ali A, Doster AR, Osorio FA: North American and European porcine reproductive and respiratory syndrome viruses differ in non-structural protein coding regions. J Gen Virol 1999, 80:307-315.

17. Shen S, Kwang J, Liu W, Liu DX: Determination of the complete nucleotide sequence of a vaccine strain of porcine reproductive and respiratory syndrome virus and identification of the Nsp2 gene with a unique insertion. Arch Virol 2000, 145:871-883.

18. Fang Y, Kim DY, Ropp S, Steen P, Christopher-Hennings J, Nelson EA Rowland RR: Heterogeneity in Nsp2 of European-like porcine reproductive and respiratory syndrome viruses isolated in the United States. Virus Res 2004, 100:229-235.

19. Feng YJ, Zhao TZ, Nguyen T, Inui K, Ma Y, Nguyen TH, Nguyen VC, Liu D, Bui QA, To LT, Wang CB, Tian KG, Gao GF: Porcine Respiratory and Reproductive Syndrome Virus Variants, Vietnam and China, 2007. Emerg Infect Dis 2008, 14:1774-1776

20. Hu HX, Li XL, Zhang ZF, Shuai JB, Chen N, Liu GQ, Fang WH: Porcine reproductive and respiratory syndrome viruses predominant in southeastern China from 2004 to 2007 were from a common source and underwent further divergence. Arch Virol 2009, 154:391-398.

21. Song J, Shen D, Cui J, Zhao BH: Accelerated evolution of PRRSV during recent outbreaks in China. Virus Genes 2010, 41:241-245

22. Tong GZ, Zhou YJ, Hao XF, Tian ZJ, An TQ, Qiu HJ: Highly pathogenic porcine reproductive and respiratory syndrome, China. Emerg Infect Dis 2007, 13:1434-1436.

23. Liu SS, Zhao YR, Hu QB, Lv CC, Zhang CF, Zhao R, Hu F, Lin WC, Cui SJ: A multiplex RT-PCR for rapid and simultaneous detection of porcine teschovirus, classical swine fever virus, and porcine reproductive and respiratory syndrome virus in clinical specimens. J Virol Methods 2011, 172:88-92.

24. Qiao Jun, Chen Chuangfu, feng Gao, Guizheng Jia: Detection of classical swine fever virus by reverse transcription polymerase chain reaction. Journal of Tarim University of Agricultural Reclamation 2001, 13:25-27.

25. Wang Xianwen, Liu Xingyou, Liang Meilan, Zheng Yushu, Liu Liyan: Detection of Porcine Circovirus Type 2 by PCR Technique. Chinese Agricultural Science Bulletin 2007, 9:28-31.

26. Li YF, Wang XL, Bo KT, Wang XW, Tang B, Yang BS, Jiang WN, Jiang P: Emergence of a highly pathogenic porcine reproductive and respiratory syndrome virus in the Mid-Eastern region of China. Vet J 2007, 174:577-584.

\section{doi:10.1186/2046-0481-65-14}

Cite this article as: Guo et al:: Outbreaks of highly pathogenic porcine reproductive and respiratory syndrome in Jiangxi province, China. Irish Veterinary Journal 2012 65:14. 